\title{
Axial penile rigidity influences patient and partner satisfaction after penile prosthesis implantation
}

\author{
Abdulla Al Ansari, Raidh A. Talib, Onder Canguven, Ahmad Shamsodini \\ Urology Department, Hamad General Hospital, Doha, Qatar
}

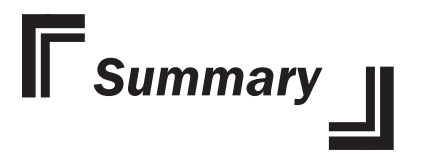

Introduction: Penile prosthesis implantation is one of the treatment choices that is kept for patients who were not satisfied with other treatments. Although penile prosthesis satisfaction rates are higher, there are some dissatisfied patients. The patients' reasons are mostly shortness and softness of implanted prosthesis. It was previously demonstrated that penile axial rigidity of more than 500 grams is enough for successful vaginal intromission. To our knowledge, there is no study comparing axial rigidity of penile prosthesis and satisfaction.

Objectives: The aim of this study was to examine whether axial rigidity of penile prosthesis had impact on patient and partner satisfaction.

Materials and Methods: We enrolled one hundred patients who were implanted penile prosthesis before to evaluate their penile axial rigidity. We used Rigidometry (by using the digital inflection rigidometer) to assess the minimal axial pressure to bend the implanted penis.

Results: We demonstrated that mean axial pressure to bend the implanted penis was $984.8 \pm 268.7$ grams. Overall satisfaction score with the penile prosthesis implant was 4.55 and 4.49 (out of 5) in patients and partners, respectively. In total, seven men were unsatisfied with their implant and reported a mean satisfaction score of $0.6 \pm 0.48$ (out of 5). All prostheses types showed good and more than 500 grams axial rigidity. The patients with Ambicor type, which were buckled at about 710.5 grams, showed worse satisfaction rates in comparison to other prostheses in two patients. Digital inflection rigidometer results of other penile prosthesis types in unsatisfied patient were 842.0, 872.0, 887.0 and 920 g. in CX700, Titan, Genesis and Titan OTR, respectively.

Conclusion: We demonstrated that dissatisfaction rate was highest in Ambicor prosthesis implanted patients. Additionally, patients with 3-piece penile prosthesis were more satisfied than 2-piece or malleable ones, interestingly, although some cases had lower axial rigidity results.

KEY WORDS: Axial buckling test; Erectile dysfunction; Penile rigidity, Satisfaction.

\section{INTRODUCTION}

Penile prosthesis (PP) is reserved for man who cannot use or fail to respond to first and second line treatments of erectile dysfunction (ED). Although majority of patients were satisfied with PP, satisfaction rates sometimes drop to 80 percent $(1,2)$. In a European study, the satisfaction rate decreased to 75 percent for specific PP types (3). According to the latter study, patients' dissatisfaction was especially related to the rigidity of PP. Comparison of the hardness of erections before and after implantation revealed that hardness varied with the dif- ferent prosthesis types; patients reported that erections were harder than before in its' natural form (3). In order to evaluate newly introduced PP, the researchers also investigated patients' satisfaction rates $(4,5)$. In most of the studies, patients were requested to fill questionnaires by phone or mail, researchers did not evaluate the patients by objective measurement techniques in addition to questionnaires $(3,6,7)$.

The aim of this study was to assess axial rigidity of six types of PP (Ambicor, CX700, Genesis, Spectra, Titan and 
Titan OTR) in 100 consecutive patients with ED. Secondary objective included assessment of patients' and partners' satisfaction with these PPs. To our knowledge this is the first report of objective and subjective PP performance by measuring their axial rigidity.

\section{MATERIAL AND METHODS}

A chart review was performed on patients that underwent PP implant surgery from January 2008 through January 2013. All surgeries were performed by the same team. All prostheses were placed through penoscrotal incision and cylinder sizes used were primarily 15-21 $\mathrm{cm}$. Enrollment and data collection were conducted at a follow-up visit at least 3 months and up to 5 years after implantation. At the follow-up visit, medical history and demographic data were collected, along with operative data and rigidity measurements. Additionally, a 10-question questionnaire from the literature (8) was used to assess satisfaction with various domains related to the PP (Table 1). The questions were designed with a Likert grading scale scored 1 through 5 (1-very unsatisfied, 2moderately unsatisfied, 3-satisfied, 4-moderately satisfied, 5 -very satisfied). Scores $\geq 3$ to the question were classified as satisfied. Answers for partner's satisfaction were attained from participants. Moreover, total score was calculated by addition of 7 questions' results (7-35). The primary efficacy endpoint for the clinical evaluation of penile rigidity by the investigators was a positive penile buckling test using the digital inflection rigidometer (DIR). Rigidometry was carried out to evaluate the minimal axial pressure to bend the implanted penis, using the DIR (H501, Electromedicina, Baleares, Spain). With the patient in a supine position, the plastic cap was applied to the tip of the penis in a downward direction by the investigator. A force of nearly $1.0 \mathrm{~kg}$ was slowly achieved on the weight scale by steadily increasing the downward force. The shaft of the penis was observed for buckling resulting from the load. Three consecutive readings of the actual axial (buckling) rigidity were averaged.

\section{Procedure}

Rigidity measurements were performed by same study investigator and technician with the DIR using the following procedure.

1. After ensuring the inflatable PP was completely deflated, the subject was asked to inflate his PP to a point where he thought it would be sufficient for sexual intercourse. (This phase was omitted in malleable PP implanted patients).

2. The investigator held the DIR in their primary hand and pushed the DIR pressure pad on the head of the penis.

3. The pressure pad was held for at least 5 seconds with a moderate pressure (500-1500 gram) or until penis buckled. The output from the DIR was recorded.

4. If penis buckled, the output from the DIR as the buckling force was recorded.

5. The investigator inflated pump when penis buckled with low pressures to see if inflation could be performed sufficiently.

\section{Stitisticd Adlysis}

Statistical package SPSS version 16.0 (Chicago, IL, USA). For continuous variables, statistics included means, standard deviations, and 95\% confidence intervals for the means when normal distribution assumptions are not violated. Comparison of the DIR and the satisfaction rate between the various PPs was performed using the Pearson chi-square test. Further patient data were obtained retrospectively from medical records. A p-value of $\leq 0.05$ was considered significant. The study was carried out with the approval of the review board of medical research center and all patients provided informed, written consent.

\section{RESULTS}

A total of hundred patients was enrolled in this study to assess the axial penile rigidity of implanted PP. Mean age of the study participants was $61.4 \pm 9.8$ years and the

Table 1.

The questionnaire administered to patients and their sexual partners (8).

\begin{tabular}{|c|c|}
\hline Questions for Patients & Responses* \\
\hline 1. How would you rate the ease of use of your PP? & $1-5$ \\
\hline 2. How would you rate the rigidity of your PP for intercourse? & $1-5$ \\
\hline 3. How satisfied are you with the length of your PP? & $1-5$ \\
\hline 4. How satisfied are you with the width of your PP? & $1-5$ \\
\hline 5. How satisfied are you with the orgasms you achieve with your PP? & $1-5$ \\
\hline 6. How satisfied do you think your sexual partner is with your PP? & $1-5$ \\
\hline 7. What is your overall satisfaction with your PP? & $1-5$ \\
\hline 8. In retrospect, would you undergo this procedure again? & Yes - No \\
\hline 9. Would you recommend this procedure to other patients? & Yes - No \\
\hline
\end{tabular}

*1-5 (1-very unsatisfied, 2-moderately unsatisfied, 3-satisfied, 4-moderately satisfied, 5-very satisfied). PP: Penile prosthesis 
Table 2.

The scores of each question and results of mean digital inflection rigidometer (DIR).

\begin{tabular}{|lcc|}
\hline & Mean & \pm Std. deviation \\
\hline Age (year) & 61.42 & 9.77 \\
\hline $1^{\text {st }} Q$ Score (1-5) & 4.82 & 0.54 \\
\hline $2^{\text {nd }}$ Q Score (1-5) & 4.55 & 1.15 \\
\hline 3 $^{\text {rd }}$ Q Score (1-5) & 4.41 & 1.39 \\
\hline $4^{\text {th }}$ Q Score (1-5) & 4.58 & 1.11 \\
\hline $5^{\text {th }}$ Q Score (1-5) & 4.67 & 1.03 \\
\hline $6^{\text {th }}$ Q Score (1-5) & 4.49 & 1.18 \\
\hline $7^{\text {th }}$ Q Score (1-5) & 4.55 & 1.09 \\
\hline Total Score of 7 questions (7-35) & 32.10 & 6.10 \\
\hline Average DIR (g) & 984.78 & 268.74 \\
\hline Minimum DIR (g) & 647.53 & 278.69 \\
\hline Maximum DIR (g) & 1275.12 & 337.83 \\
\hline Sexual Intercourse frequency/month & 9.18 & 5.31 \\
\hline
\end{tabular}

common causes of ED were diabetes mellitus (74), atherosclerotic disease (18), and radical surgery (8).

Only 8 patients had Peyronie's disease. Mean follow-up was 7 months (range: 3-60). The results of each question were given in Table 2 .

Types of the implants and unsatisfaction rates were described in Table 3. Mean male and female satisfaction with the PP implant was 4.55 and 4.49 (out of 5), respectively. Interestingly, nearly one third (28/100) of patients admitted that their wives do not know that they had PP. Overall, seven men were unsatisfied with their PP implant and reported a mean satisfaction score of $0.6 \pm 0.48$ (out of 5). PP types of unsatisfied patients and the rates according to the same type implantation were Ambicor (3/15), CX700 (1/42), Genesis (1/9), Titan (1/13), Titan OTR (1/15) (Table 3).

Average DIR of all types of PP was $984.8 \pm 268.7$ g. In three patients, investigator inflated pump when penis buckled with low pressures and found that the inflation was not sufficiently obtained by the patients. The mean age of these patients were $74 \pm 1.33$ years. We found DIR results of $\mathrm{PP}$ in unsatisfied patient as 710.5, 842.0, 872.0, 887.0 and 920 g. in Ambicor, CX700, Titan, Genesis and Titan OTR, respectively. Especially, DIR results of 3 Ambicor PP that were implanted 5 years ago were significantly lower than the other satisfied patients' average DIR. In general, main reasons of dissatisfaction were hardness and shortness of penis.

For retrospective question (\#8), 89 patients responded that they would undergo this procedure again. For recommendation question (\#9), 91 patients responded that they would recommend this procedure to other patients. The average of PP usage for sexual activity was 9.18 \pm 5.31 per month. Pearson's correlation analysis suggested a direct correlation between answers for questions \#2 and $\# 7$ ( $p<0.01 ; r=0.723)$. There was a significant correlation between average DIR and overall satisfaction $(p<0.05 ; r=0.232)$. Interestingly, there was no statistically significant relation between total score and penile length and width (Table 4).
Table 3.

Mean digital inflection rigidometer (DIR) and dissatisfaction percentages of six type of penile prosthesis.

\begin{tabular}{|c|c|c|c|c|}
\hline \multirow{4}{*}{$\begin{array}{l}\frac{0}{d} \\
\frac{d}{d} \\
\frac{\sigma}{\Phi} \\
\underline{\Xi}\end{array}$} & Types of prostheses & $\underset{\text { (implanted) }}{\mathbf{N}}$ & $\begin{array}{c}\mathrm{n} / \mathrm{N}(\%) \\
\text { (unsatisfied) }\end{array}$ & Mean DIR \\
\hline & CX700 ((3-Piece, AMS) & 42 & $1 / 42(2.4 \%)$ & 985 \\
\hline & Ambicor (2-Piece, AMS) & 15 & $3 / 15(20 \%)$ & 870 \\
\hline & Titan (3-Piece, Coloplast) & 13 & $1 / 13(7.7 \%)$ & 1068 \\
\hline \multirow{4}{*}{$\frac{\mathbb{\mathscr { d }}}{\frac{\mathbb{\pi}}{\sum}}$} & Titan OTR (3-Piece, Coloplast) & 15 & $1 / 15(6.6 \%)$ & 953 \\
\hline & Genesis (Coloplast) & 9 & $1 / 9(11.1 \%)$ & 857 \\
\hline & Spectra (AMS) & 6 & - & 837 \\
\hline & $\overline{\text { Total }}$ & 100 & $7 / 100(7 \%)$ & 984.8* \\
\hline
\end{tabular}

* There was no statistically difference between mean digital inflection rigidometer of all types (984.8 grams) and each type of penile prosthesis ( $p>0.05$ ). (AMS: American Medical System; OTR: one touch release).

Table 4.

Correlation between the total score of 7 questions (32.1 \pm 6.1 ) with digital inflection rigidometer, penile length and penile width, provided by Pearson correlation analysis.

\begin{tabular}{|lccc|}
\hline & Average digital inflection rigidometer & Penile length & Penile width \\
\hline Total score & $p=0.022 *$ & $p=0.252$ & $p=0.146$ \\
\hline
\end{tabular}

${ }^{\star} p$-value of $\leq 0.05$ was considered significant.

\section{Discussion}

The PP implantation is one of the modality of treatment with high success rate for ED. Although axial rigidity objectively defines the capability of the PP to resist buckle during vaginal intromission, there is no study specifically assessing axial rigidity of implanted PP. This study extends current knowledge in the satisfaction of PP by further examining the axial rigidity of the different types of PPs. In this study, we demonstrated that five different PP types other than Ambicor PP had good axial rigidity that is needed for a successful intercourse. We showed that 95\% male participants were satisfied with PP implantation. The history of surgical implantation for ED was first recorded in 1930s by Bogoras, who used a tailored section of rib cartilage to create the os penis of animals and produce rigidity in a reconstructed penis (9). Today PPs can broadly be divided into malleable and inflatable ones. Since PP implants are associated with a high level of patient satisfaction, researchers always investigate and compare firstly introduced PP with the previous types from different points of satisfaction $(3,4,6)$. Axial and radial rigidity of penis share a common dependency upon intracavernosal pressure. However, axial rigidity, not radial penile 
deformation, is the physical parameter which best defines the capability of the erect penis to resist buckle during vaginal intromission, and pelvic thrusting following penetration (10). The penile buckling force classically measures axial rigidity. Axial rigidity assessment was introduced during the early 1980s at some stage in sleep laboratory research and remains a simple and inexpensive diagnostic tool (11). Later, during the evaluation of efficacy of interventions for ED, researchers used and recommended axial rigidity parameter in their studies $(10,12-14)$. Karacan et al. demonstrated that force on a rod less than 500 gram (g.) were unable to achieve intromission in any female subject (11). Based on Karacan's study, it has been assumed that $500 \mathrm{~g}$. axial force is the minimum pressure necessary for vaginal penetration. In our study, mean average DIR for all types of prosthesis was about $985 \mathrm{~g}$. that is sufficient for intromission. On the other hand, although our unsatisfied patients' DIR results were above $500 \mathrm{~g}$. they were less than the average. According to our results, axial rigidity, which is nearly $1000 \mathrm{~g}$. by DIR, is necessary for PP implanted patients' satisfaction.

In our study, we found that dissatisfaction rate was highest in Ambicor PP implanted patients. Significantly, DIR results were almost 30\% less in unsatisfied Ambicor implanted patients than the average DIR. Ambicor is a 2piece inflatable PP which was introduced in 1994 and underwent reinforcement of the pump tubing connection to decrease fluid leak failure in 1998 (5).

Unfortunately, one of our Ambicor unsatisfied patient also revealed spontaneous deflation during intercourse, which occurred 5 years after the implantation. In a multicentre study, examining 3 different PP, Ambicor was found as the less hard than the other two PP (3). In literature, the ratio of mechanical failure is found in between $0.7 \%$ to $15 \%$ with the Ambicor PP $(3,5)$. Previosuly, less hardness of Ambicor was connected to the limited volume of fluid transferred out of the cylinders in the Ambicor (5).

Although PP is the last option in treatment of ED, almost all the patients and their partners were satisfied after PP implantation $(3,4,6)$. As has been found in the previous study, PP has the second highest satisfaction rate after oral medications among other treatment modalities e.g. intracavernosal injections and vacuum device (15). In order to describe satisfaction and partner sexual function after PP implantation, Moskovic et al. designed a survey to assess various aspects of patient and partner satisfaction related to their PP (8). According to their study results, partner satisfaction scores were higher, respectively, in men with higher PP satisfaction than those with lower PP (8). Studies, which are examining satisfaction of patients and partners at the same time, offer us both objective and subjective satisfaction rates. The more impressive functional results of studies investigating both patients and partners are that the results are mostly in close proximity to each other $(4,8)$. It was demonstrated that both patients and their female partners report high levels of satisfaction several years after inflatable PP implantation (6). We found very high satisfaction rates for both patients and partners. This result is consistent with literature about this subject $(3,4,6)$.
In a European study, Natali et al. reported a satisfaction in $97 \%$ of patient who underwent PP implantation and in $91 \%$ of their partners (3). The results of the present study showed that majority (89\%) of our study participants responded that they would undergo PP procedure again. We believe that this high rate positive answer for the latter has important clinical implication from point of satisfaction.

An important observation of our study was that three of our study patients could not inflate the PP until the requested rigidity. This was mostly due to the fact that they evaluate the degree of rigidity obtained was enough good for penetration. There is no available data in the literature on the number of squeezes necessary to get enough hardness of erection, because of variability of volumes needed in relation to the patient's penis size. Although the number of patients who could not inflate PP properly was minor in this study, we propose that this point should be kept in mind in differential diagnosis of patients' complain for reduced hardness of PP, especially in elder people. On the other hand, 3-piece inflatable PPs (AMS CX700 and Titan OTR) demonstrated good results in terms of rigidity measured by DIR.

Additionally, these 3-piece inflatable PPs also did not show any mechanical failure in long-term follow up of in our study participants. A recent study conducted by Bernal and Henry who reviewed last 20 years' articles that included more than 30 patients showed that patients with ED who underwent 3-piece PP placement reported the highest satisfaction rates (16).

The present study was designed with the purpose of assessing axial rigidity in association with patients and partners satisfaction. To our knowledge, this is the first study evaluating PP by axial rigidity. However, the present study might have some limitations. A limitation of this study is that we could not apply our satisfaction questions directly to the partners. This was mainly because of culture difference in our region that our study patients did not want us to speak with their wives on this subject. It should be pointed out that the patient-reported partner satisfaction might have been overestimated because of this reason. However, regarding partner satisfaction, we surprisingly discovered that nearly one-third our patients' partner do not have any knowledge about their spouses' prosthesis. A further limitation is the difference in the numbers of different PP types evauated. Since we implant 3-piece inflatable PP more than the others, there was a big discrepancy in numbers, which could not be avoided.

\section{Conclusion}

Using DIR, we demonstrated that five different PP types have good axial rigidity for successful intercourse with high patient and partner satisfaction. DIR results of 2piece Ambicor PP were significantly lower than the other PP especially in dissatisfied patients. Patient and partner satisfaction rates were roughly similar to those reported in the literature. We believe that reporting specific data for different implant types with axial rigidity for patient and partner satisfaction is significant for the future researches. 


\section{ACKNOWLEDGEMENTS}

A grant from the Hamad Medical Corporation primarily supported this research.

We would also like to acknowledge the careful work of Dr. Abdulbari Benar for his assistance with the statistics used in this study and also our technician Mr. Ahmed Sandly for his help while using rigidometer.

\section{REFERENCES}

1. Porena M, Mearini L, Mearini E, et al. Penile prosthesis implantation and couple's satisfaction. Urol Int 1999; 63:185-7.

2. Mulhall JP, Ahmed A, Branch J, Parker M. Serial assessment of efficacy and satisfaction profiles following penile prosthesis surgery. J Urol. 2003; 169:1429-33.

3. Natali A, Olianas R, Fisch M. Penile implantation in Europe: successes and complications with 253 implants in Italy and Germany. J Sex Med. 2008; 5:1503-12.

4. Jensen JB, Madsen SS, Larsen EH, et al. Patient and partner satisfaction with the Mentor Alpha-1 inflatable penile prosthesis. Scand J Urol Nephrol. 2005; 39:66-8.

5. Lux M, Reyes-Vallejo L, Morgentaler A, Levine LA. Outcomes and satisfaction rates for the redesigned 2-piece penile prosthesis. J Urol. 2007; 177:262-6.

6. Bettocchi C, Palumbo F, Spilotros M, et al. Patient and partner satisfaction after AMS inflatable penile prosthesis implant. J Sex Med. 2010; 7:304-9.

7. Carson CC, Mulcahy JJ, Govier FE. Efficacy, safety and patient satisfaction outcomes of the AMS 700CX inflatable penile prosthesis: results of a long-term multicenter study. AMS 700CX Study Group. J Urol. 2000; 164:376-80.

8. Moskovic DJ, Gittens P, Avila D Jr., et al. Favorable female sexual function is associated with patient satisfaction after inflatable penile prosthesis implantation. J Sex Med. 2011; 8:1996-2001.

9. Kaneko S, Mizunaga M, Yachiku S, et al. Clinical applicability of a new tactile sensor for evaluating rigidity of the penis: a comparative study with Rigiscan. Int J Urol. 1996; 3:379-82.

10. Udelson D, Park K, Sadeghi-Nejad H, et al. Axial penile buckling forces vs Rigiscan radial rigidity as a function of intracavernosal pressure: why Rigiscan does not predict functional erections in individual patients. Int J Impot Res. 1999; 11:327-37; discusion 37-9.

11. Karacan I, Moore CA, Sahmay S. Measurement of pressure necessary for vaginal penetration. Sleep Res. 1985; 14:269-72.

12. Henry GD, Jennermann C, Eid JF. Evaluation of satisfaction and axial rigidity with Titan XL cylinders. Adv Urol. 2012; 1-6.

13. Sidi AA, Lange PH. Recent advances in the diagnosis and management of impotence. Urol Clin North Am. 1986; 13:489-500.

14. Goldstein I, Auerbach S, Padma-Nathan H, et al. Axial penile rigidity as primary efficacy outcome during multi-institutional inoffice dose titration clinical trials with alprostadil alfadex in patients with erectile dysfunction. Alprostadil Alfadex Study Group. Int J Impot Res 2000; 12:205-11.

15. Hassan A, El-Hadidy M, El-Deeck BS, Mostafa T. Couple satisfaction to different therapeutic modalities for organic erectile dysfunction. J Sex Med. 2008; 5:2381-91.

16. Bernal RM, Henry GD. Contemporary patient satisfaction rates for three-piece inflatable penile prostheses. Adv Urol. 2012; 1-5.

\section{Correspondence}

Abdulla Al Ansari, MD

Associate Professor

Raidh A. Talib, MD

Urology Department

Onder Canguven, MD (Corresponding Author)

Associate Professor

ocanguven@yahoo.com

Ahmad Shamsodini, MD

Urology Department

Urology Department, Hamad General Hospital

Hamad Medical Corporation

3050, Doha, Qatar 